\title{
Substrate specificity of the ubiquitin and Ubl proteases
}

\author{
Judith A Ronau ${ }^{1}$, John F Beckmann ${ }^{1}$, Mark Hochstrasser ${ }^{1,2}$ \\ ${ }^{I}$ Department of Molecular Biophysics and Biochemistry, ${ }^{2}$ Department of Molecular, Cellular and Developmental Biology, Yale \\ University, 266 Whitney Avenue, New Haven, CT 06520, USA
}

Conjugation and deconjugation of ubiquitin and ubiquitin-like proteins (Ubls) to cellular proteins are highly regulated processes integral to cellular homeostasis. Most often, the C-termini of these small polypeptides are attached to lysine side chains of target proteins by an amide (isopeptide) linkage. Deubiquitinating enzymes (DUBs) and Ubl-specific proteases (ULPs) comprise a diverse group of proteases that recognize and remove ubiquitin and Ubls from their substrates. How DUBs and ULPs distinguish among different modifiers, or different polymeric forms of these modifiers, remains poorly understood. The specificity of ubiquitin/Ubl-deconjugating enzymes for particular substrates depends on multiple factors, ranging from the topography of specific substrate features, as in different polyubiquitin chain types, to structural elements unique to each enzyme. Here we summarize recent structural and biochemical studies that provide insights into mechanisms of substrate specificity among various DUBs and ULPs. We also discuss the unexpected specificities of non-eukaryotic proteases in these families.

Keywords: ubiquitin; DUBs; Ubl proteases; SUMO; Nedd8; ULPs

Cell Research (2016) 26:441-456. doi:10.1038/cr.2016.38; published online 25 March 2016

\section{Introduction}

Covalent attachment of ubiquitin to proteins governs a wide array of cellular processes, including cell division, DNA repair, endocytosis, cellular signaling, and protein quality control [1-4]. The sequential action of three enzymes - E1 ubiquitin-activating enzyme, E2 ubiquitin-conjugating enzyme, and E3 ubiquitin ligase - results in attachment of ubiquitin to a substrate protein, usually via an amide (isopeptide) bond that links the C-terminal carboxyl group of ubiquitin with a lysine side chain(s) of the protein substrate (Figure 1) [5, 6]. Ubiquitin itself possesses seven lysine residues (Lys6, $11,27,29,33,48$, and 63), enabling it to form ubiquitin polymers; chains with different linkages signal different functional outcomes for the tagged proteins [3, 7-9]. All seven lysines are used for chain formation in vivo, as is the N-terminal $\alpha$-amino group, the latter leading to 'linear' ubiquitin chains. Chains can be homopolymeric or, less commonly, of mixed linkage. Branched ubiquitin chains, which use different lysines of a single ubiquitin for chain extension, can also form [10-13].

Correspondence: Mark Hochstrasser

E-mail: mark.hochstrasser@yale.edu

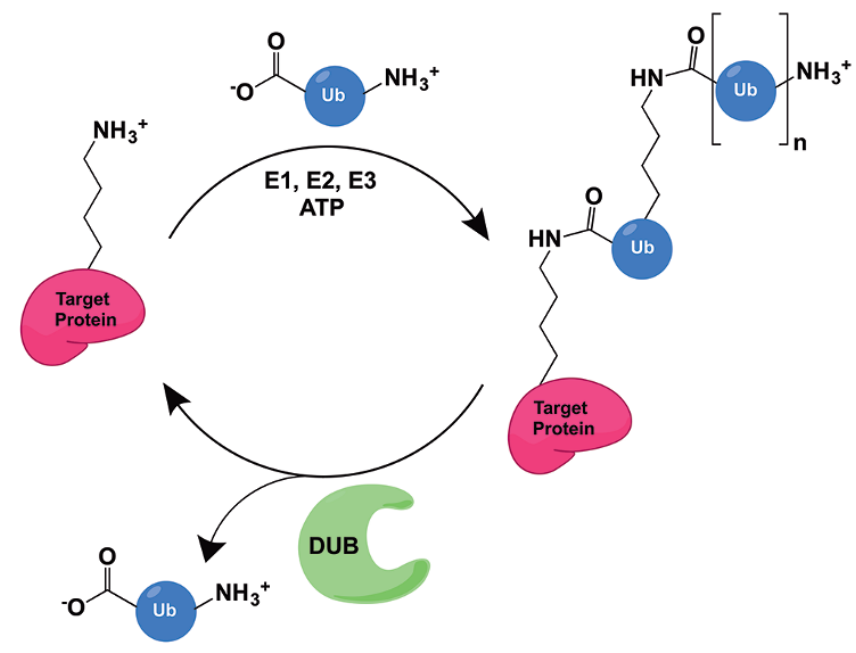

Figure 1 The ubiquitin (Ub) modification cycle.

Following the discovery of ubiquitin, related proteins called ubiquitin-like proteins (Ubls) were also identified; these proteins share a common core architecture called the $\beta$-grasp fold [14]. As with ubiquitin conjugation, a similar cascade of E1, E2 and E3 enzymes, specific to each Ubl, is utilized to covalently link Ubl and substrate [15]. Besides ubiquitin, the most frequently employed 
Ubl is SUMO ( small ubiquitin-related modifier). In humans, SUMO is present as four isoforms, each encoded by a distinct gene [16]. Interestingly, hybrid SUMO-ubiquitin chains have also been described [17]. The variety of covalently ligated ubiquitin/Ubls and their polymeric forms creates significant challenges for the deconjugating enzymes in identifying and cleaving the appropriate substrates.

Deubiquitinating enzymes (DUBs) and Ubl-specific proteases (ULPs) catalyze the cleavage of ubiquitin or Ubls from substrate proteins and also process C-terminally extended precursor forms of these modifiers (Figure 1) [18]. DUBs and ULPs can be classified into one of two mechanistic classes: (1) thiol proteases, which are mechanistically and structurally related to the well-studied cysteine protease papain and rely on a nucleophilic cysteine in the active site for catalysis, and (2) metalloproteases, which coordinate a $\mathrm{Zn}^{2+}$ ion in the active site and use a nucleophilic water ligated to the metal to hydrolyze the isopeptide linkage [19]. The JAB1/MPN/ MOV34 metalloproteases (JAMMs) include multiple DUBs and at least one ULP (a deneddylase, i.e., an enzyme that cleaves the Nedd8 Ubl from substrates). The thiol protease class includes the great majority of DUBs and ULPs. They are categorized into distinct families according to the structure of their catalytic domains. As with the JAMMs, the same family may have members that cleave ubiquitin, Ubls, or both. It remains difficult to predict these specificities based on enzyme primary sequence alone.

The four known eukaryotic thiol protease DUB families are the ubiquitin C-terminal hydrolases (UCHs), ubiquitin-specific proteases (USPs), ovarian tumor (OTU) proteases, and Machado-Josephin-domain proteases [20, 21]. As will be discussed later, bacterial and viral thiol proteases outside of these families have been found to have DUB activity; it is noteworthy that these bacterial and viral ubiquitin-cleaving enzymes all function within eukaryotic cells. Similar to the DUBs, there are multiple SUMO-specific thiol protease families: the SUMO/ sentrin-specific proteases (SENPs; which are related to yeast Ulp1), desumoylating isopeptidases (DESIs), and ubiquitin-specific protease-like 1 (USPL1) [16]. SUMO proteases of the SENP/ULP class are the most phylogenetically widespread.

Nearly 100 DUBs have been identified in humans. Since the capacity for ubiquitin deconjugation in cells is extremely high, these activities must be kept under tight control. Accordingly, DUB (and ULP) activity is regulated by a host of factors. In many cases, DUBs and ULPs have domains extending from either end of their catalytic domains that help regulate their activity. Such regulation may occur by facilitating enzyme-cofactor interactions, targeting enzymes to specific cellular compartments, maintaining enzymes in an auto-inhibited state, and/or altering their affinity for substrate [20].

DUBs and ULPs display high specificity toward their substrates. They can differentiate between ubiquitin and Ubls, show preferences for particular polymeric forms of ubiquitin or Ubls, and distinguish among distinct conjugated substrates. The molecular basis of this specificity is the subject of the present review. We focus on revelations about specificity derived from recent structural studies. We also discuss non-eukaryotic DUBs and ULPs and their sometimes surprising specificities.

\section{Ubiquitin and Ubl recognition by DUBs and ULPs}

\section{Modifier properties}

The modifier proteins sport surface features that aid in their recognition by DUBs and ULPs. Although ubiquitin is a small, compact protein with a rigid core, it contains several important motifs for interactions with other proteins. The two motifs most commonly observed contacting DUBs are the so-called Ile44 patch (comprising Ile44, Leu8, Val70 and His68) and the Ile36 patch (Ile36, Leu71 and Leu73) $[22,23]$. Other protein-binding elements utilized by ubiquitin are the Phe 4 patch (Gln2, Phe 4 and Thr14), the TEK box (Lys6, Lys11, Thr12, Thr14 and Glu34), and the Asp58 patch (Arg54, Thr55, Ser57 and Asp58) [3].

Sequence alignment of ubiquitin and the Ubls SUMO, Nedd8, ISG15 and Fat10 reveals that, aside from Nedd8, the other Ubls exhibit very little sequence conservation with ubiquitin in these motifs (Figure 2). Both the Ile36 and Ile44 patches are conserved in Nedd8, and the Nedd8 Ile44 patch binds directly to the deneddylase Den1/ SENP8 [24, 25]. However, the Ile44 patch is not always a key DUB/ULP contact spot. SdeA, a bacterial DUB, does not engage in any interactions with this patch when bound to a ubiquitin suicide substrate [26]. Likewise, new crystal structures of the USP CYLD bound to either Met1 - or Lys63-linked diubiquitin revealed that the Ile44 patch of the distal ubiquitin (the one with its $\mathrm{C}$-terminal carboxyl in amide linkage) has no direct interactions with the enzyme [27]. A similar observation was made previously for USP7 bound to ubiquitin aldehyde [28].

Perhaps the most significant feature of ubiquitin and Ubls for cognate protease recognition is their flexible C-terminal tail [29]. The ubiquitin/Ubl tail is stabilized by several interactions in the protease active site cleft. DUB and ULP deconjugation of their cognate modifier proteins depends heavily on these $\mathrm{C}$-terminal residues (labeled P6-P1 in Figure 2). For example, a single ami- 
$\mathbf{A}$
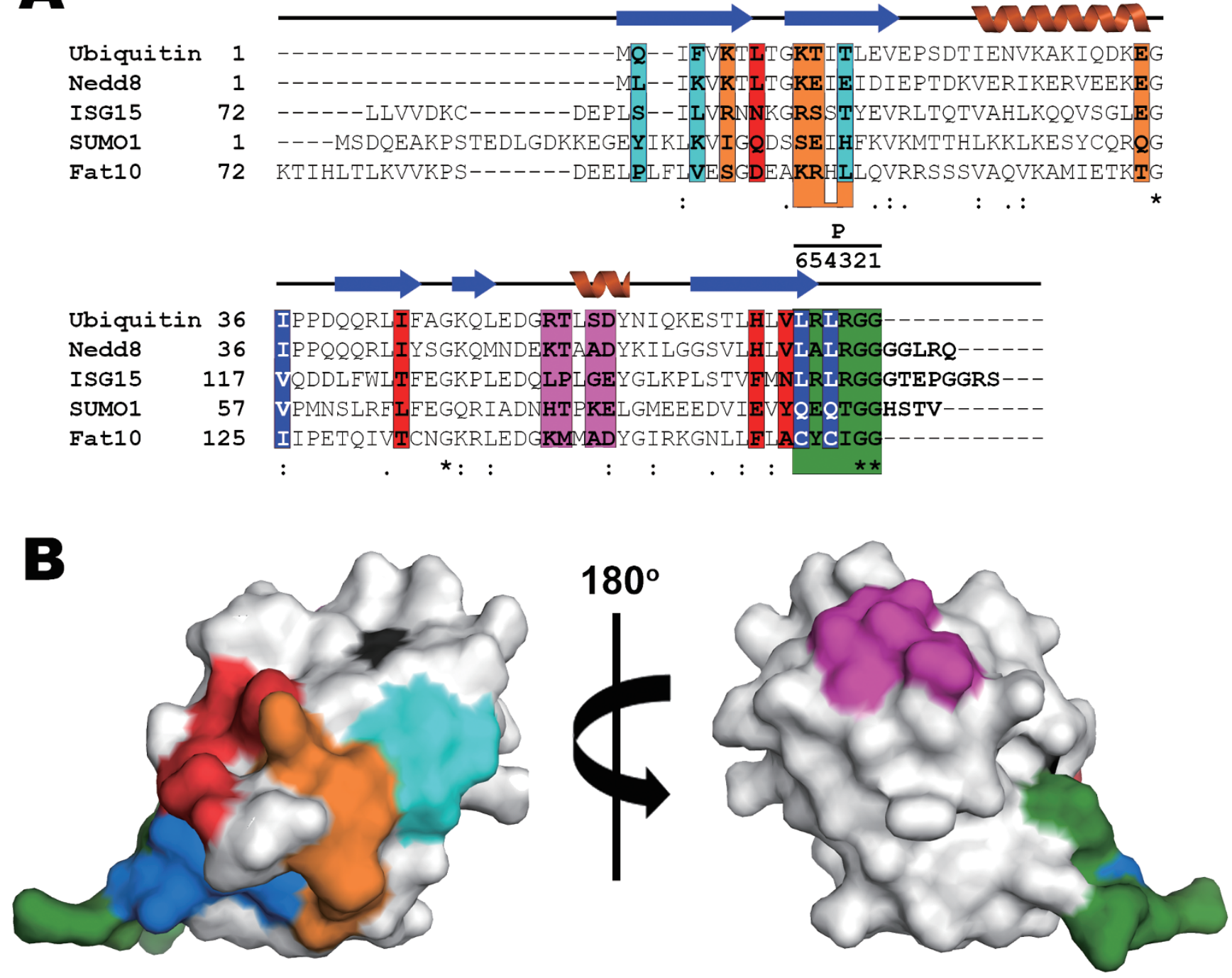

Figure 2 Sequence alignment of ubiquitin and Ubls (A) and ubiquitin surface elements important for protein binding (B). The various patches that ubiquitin (PDB code: $1 \mathrm{UBQ}$ [31]) uses to bind other proteins are highlighted as follows: the C-terminal LRLRGG motif is colored green, the lle44 patch is red, the lle36 patch is blue, the Phe4 patch is cyan, the TEK box is orange, the Asp58 patch is purple, and Ser65, which is phosphorylated by the kinase PINK1, is black.

no-acid exchange of the Ala at position P5 in Nedd8 to an Arg, which is the ubiquitin P5 residue, markedly decreased the affinity of Den1 for the mutated Ubl, likely due to steric interference with the ULP [25]. Conversely, for USP21, which exhibits dual specificity for ubiquitin and ISG15, Arg72 at P5 of ubiquitin is stabilized through formation of a salt bridge with an invariant Glu in USP21 [30]. This Arg residue is present in the sequence of ISG15 but not Nedd8. Were Arg72 not engaged in DUB binding, one might predict that discrimination against Nedd8 would not be seen; this is borne out for the prokaryotic DUB SdeA [26].
Other ubiquitin/Ubl features also contribute to binding of their cognate deconjugating enzymes. In the $\beta$-grasp fold, a central $\alpha$-helix is cradled by a curved $\beta$-sheet (Figure 3) [31]. Most co-crystal structures of ubiquitin-DUB complexes reveal that the two-residue loop (Leu8-Thr9) that connects the $\beta 1$ and $\beta 2$ strands nestles into a binding pocket in DUBs well away from the active site [28, 32, 33]. Binding by this loop in Nedd8 also generates several key van der Waals contacts with the ULP Den1 [24, 25]. By contrast, inspection of co-crystal structures of SUMO with SUMO proteases indicates that the SUMO $\beta 1-\beta 2$ loop has little direct involvement in binding to the prote- 

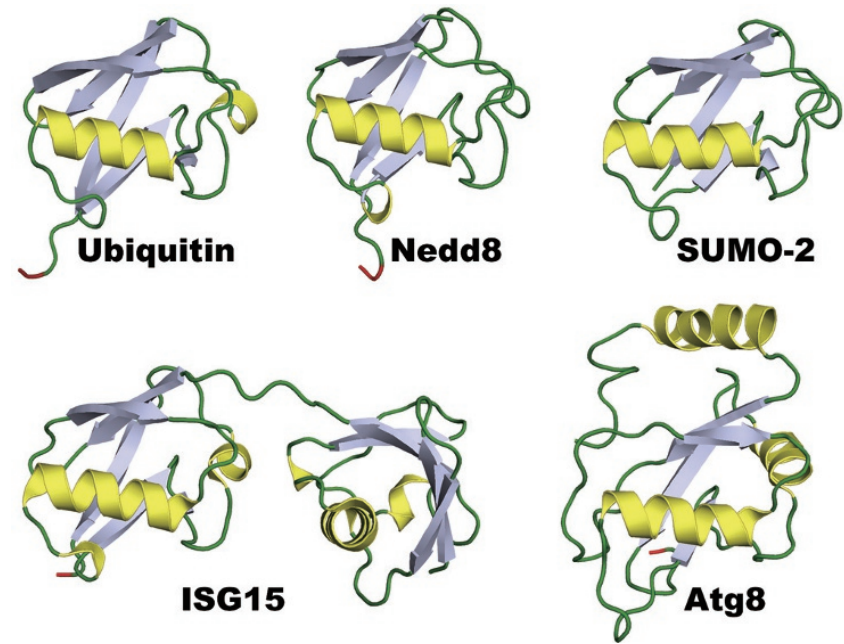

Figure 3 Structural comparison depicting the conserved $\beta$-grasp fold of ubiquitin and Ubl proteins (PDB codes: 1UBQ [31], 1NDD [146], 1WM3 [147], 1Z2M [148], and 2KWC [149]). ISG15 has tandem ubiquitin folds. The C-terminal glycine of ubiquitin and Ubls is colored red, except for SUMO2 because it was not ordered in the crystal structure.

ases [34-36]. Interestingly, ubiquitin co-crystal structures with UCH and USP family DUBs suggest that this ubiquitin loop adopts UCH-specific and USP-specific conformations [37].

A newly identified feature of ubiquitin, that it can be phosphorylated at Ser65 by PINK1 [38, 39], provides an added element to consider in substrate specificity. Although yeast lacks an ortholog for PINK1, phosphorylation of ubiquitin Ser65 has also been shown in yeast and may serve a conserved regulatory function [40]. Phosphorylated ubiquitin can adopt two states in solution; one may limit accessibility of its tail [41]. Most DUBs tested so far have only weak activity toward ubiquitin chains composed of phosphorylated ubiquitin [41, 42]. Ser65 is conserved in both ISG15 and Nedd8, suggesting that they may also be susceptible to phosphorylation.

\section{Recognition of polyubiquitin chains}

For depolymerization of polyubiquitinated substrates, an important structural feature is the isopeptide linkage between ubiquitin monomers. The isopeptide bond linking the proximal (lysine-donating) and distal (lysine-accepting) ubiquitin must be stabilized in the active site of the DUB. Comparison of Lys63- and Lys48-linked polyubiquitin chains shows that Lys63 linkages adopt an extended 'beads-on-a-string' conformation in which the only interaction between the ubiquitin moieties is through the isopeptide linkage [43, 44]. This conformation is shared by Met1-linked diubiquitin [45]. By contrast, the ubiquitin moieties in Lys48-linked polyubiquitin pack closely in a closed conformation through interactions of their Ile44 patches [46, 47]. Similarly, Lys11- and Lys6-linked diubiquitins also adopt more compact conformations, although the Ile44 patch is exposed on both ubiquitins in Lys11-linked diubiquitin and one of the ubiquitins in Lys6-linked diubiquitin [4850]. Recent structural studies revealed that K33-linked ubiquitin chains adopt open and closed conformations in triubiquitin and diubiquitin, respectively [51]. Nevertheless, polyubiquitin chains are dynamic in solution, and a chain can adopt both closed and open conformations [52, 53].

Compact chains likely cannot be recognized by DUBs unless they undergo significant conformational changes to expose the isopeptide bond. In the case of Lys48-linkages, this remodeling probably also involves exposure of the Ile44 patch so that it is free to interact with the DUB. To our knowledge, no DUB bound to a Lys48-linked diubiquitin has been crystallized. Specificity of DUBs toward different ubiquitin linkages varies among family members. Many JAMM proteases, such as AMSH, are only active against Lys63-linked chains [54, 55], primarily due to interactions with the proximal ubiquitin moiety $[33,56]$. On the other hand, most DUBs belonging to the USP family will hydrolyze many chain types, albeit with different preferences $[20,57]$. The OTU DUBs show a striking range of chain linkage preferences. Structural analyses reveal that proper positioning of the proximal ubiquitin on the OTU DUB surface is paramount for this selectivity [58] and that the length of the ubiquitin polymer also contributes to specificity $[58,59]$.

\section{Ubiquitin/Ubl-induced active-site rearrangement}

Thiol protease DUBs and ULPs utilize variations of the classic papain-like Cys-His-Asp/Asn catalytic triad to catalyze hydrolysis of peptide or isopeptide bonds [18]. To facilitate activation of the nucleophilic Cys by His (serving as the general base), these residues must be precisely oriented in the active site with a His-Cys hydrogen-bond distance within $\sim 3.8 \AA$. However, a common theme emerging from structural studies of these proteases is that the catalytic residues are often in unproductive orientations in the absence of substrate. This misalignment involves displacement of the Cys, the His, or both [28, 60-64]. Substrate binding causes the active site residues to rearrange into a catalytically competent orientation [32, 63, 65-67]. The earliest example of such a substrate-induced realignment of active site residues was the DUB USP7, which has been discussed extensively [20, 28, 68]. 
A striking example of how remote substrate binding induces realignment of the active-site His to a catalytically productive orientation can be seen with the free and ubiquitin-bound forms of UCHL1. Defects in UCHL1, the smallest member of the UCH family of DUBs, have been linked to a variety of diseases including Parkinson's disease and various cancers $[69,70]$. In the substrate-free form, the active site His has rotated away from the nucleophilic Cys into a catalytically unproductive orientation, and the two residues are separated by $7.7 \AA[60]$ (Figure $4 A)$. Docking of the $\beta 1-\beta 2$ hairpin loop of ubiquitin into a surface-exposed hydrophobic pocket of UCHL1 located $17 \AA$ from the catalytic triad elicits a cascade of conformational changes in highly conserved Phe residues that bridge the distal and active sites. To accommodate the $\beta$-hairpin loop of ubiquitin, UCHL1-Phe213 swings inward into the site normally occupied by Phe53, which, in turn, forces the aromatic side chain of Phe53 to rotate into a steric conflict with the catalytic His161. This causes the His side chain to flip towards the nucleophilic Cys such that the $\mathrm{N} \delta 1$ atom of the imidazole ring is now $3.9 \AA$ away from the sulfur atom of Cys90 [65]. These results suggest that interactions with the substrate outside of the DUB active site can contribute to discrimination of ubiquitin from Ubl modifiers (Figure 2A).

OTULIN (Fam105b, gumby) is a member of the OTU family of DUBs known to play roles in innate immune and Wnt signaling, as well as to bind and regulate the linear ubiquitin assembly complex (LUBAC). OTULIN harbors a Cys-His-Asn triad and only cleaves Met1linked polymers $[63,71,72]$. A high-resolution crystal structure of the apo form of OTULIN revealed mixed

Figure 4 The active site residues of unliganded Ubl proteases (shown in white) are often misaligned, but undergo restructuring into productive conformations upon substrate binding (residues from ubiquitin-bound DUBs are colored green). (A) Binding of the $\beta 1-\beta 2$ hairpin loop of ubiquitin (ubiquitin is colored blue while the loop is magenta) into a hydrophobic pocket on the surface of UCHL1 triggers a series of conformational changes in aromatic side chains, forcing His161 to adopt a productive orientation (PDB codes: 2ETL [60], 3KW5 [65]). (B) The apo structure of OTULIN reveals that $\mathrm{H} 339$ is stabilized in an unproductive form by a salt bridge with a neighboring Asp residue (PDB code: 3ZNV [63]). Binding of Met1-linked diubiquitin in the active site of OTULIN imposes a steric clash (magenta dashes) with M1 and E16 of ubiquitin, forcing $\mathrm{H} 339$ to flip into the active site (PDB code: 3ZNZ [63]). (C) Binding of Met1-linked diubiquitin leads to stabilization of $\mathrm{H} 801$ in the active site via Q2 of the proximal ubiquitin (PDB codes: 2VHF [73], 3WXE [27]). However, the Gly-Gly motif (magenta) is displaced $8 \AA$ from the active site due to E674 from a loop that is stabilized by substrate binding, which appears to block the active site.
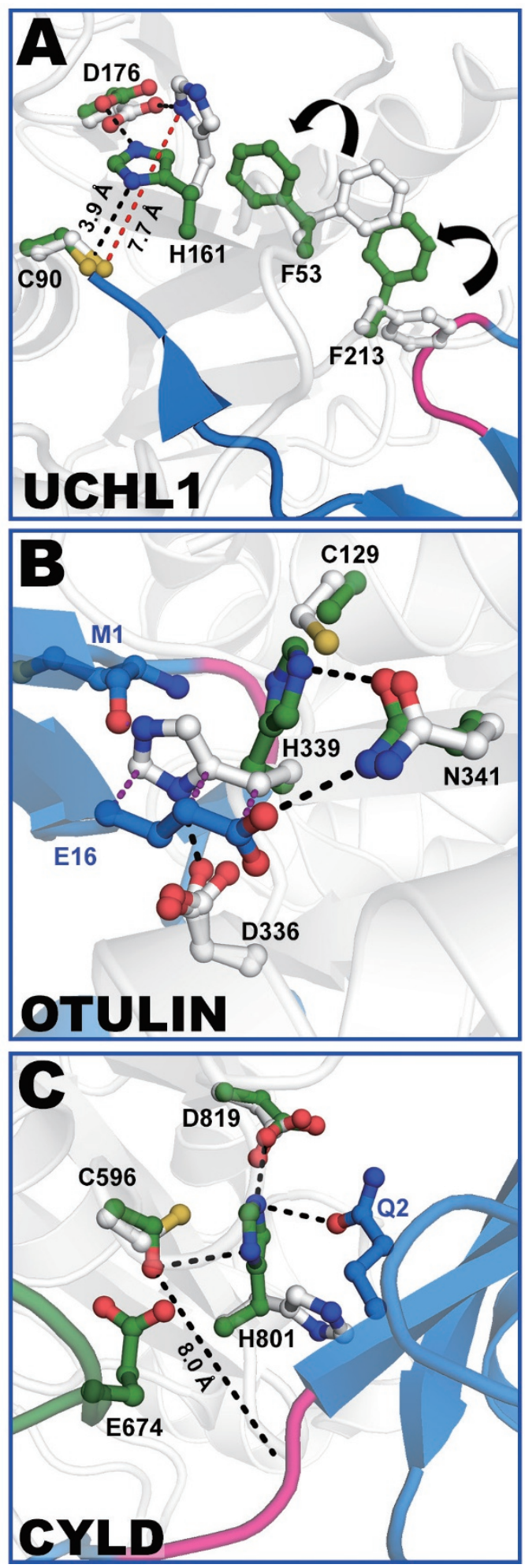

occupancy of the His and Cys catalytic residues in which $70 \%$ of the time, the residues are misaligned in an auto-inhibited state. A local non-catalytic Asp residue plays an inhibitory role by interacting with the catalytic His 
and pulling it out of hydrogen-bonding distance with the catalytic Cys (Figure 4B). Substrate-assisted realignment of the OTULIN active site into a productive form is facilitated by two unique structural features of Met1-linked diubiquitin $[63,72]$. First, the unproductive orientation of the His residue would sterically clash with the Met1 carbonyl group in the proximal ubiquitin moiety of bound linear diubiquitin. Binding of this diubiquitin forces the His residue to assume a productive orientation (Figure 4B). Isopeptide ubiquitin linkages would not induce such a change. A second key to substrate-induced activation is the positioning of Glu16 of the proximal ubiquitin, which both expels the auto-inhibitory Asp from contact with the active site His and hydrogen bonds with the Asn of the catalytic triad to align it within hydrogen-bonding distance of the His residue [63], thus forming a catalytically competent active site.

CYLD is a tumor suppressor that belongs to the USP family of DUBs and displays specificity for hydrolysis of both Met1- and Lys63-linked ubiquitin chains [45, 73]. It was crystallized in its ligand-free form with two molecules in the asymmetric unit, in which the active site residues in one molecule were oriented in a productive conformation, while in the other molecule, the side chain of the catalytic His residue was rotated away from the Cys nucleophile (Figure 4C) [73]. Recent structural characterization of CYLD bound to Lys63-linked diubiquitin in the catalytic state and Met1-linked diubiquitin in both the pre-catalytic and catalytic states revealed that the His side chain is arranged in a catalytically competent orientation with either diubiquitin [27]. However, no evidence of substrate-induced conformational crosstalk was seen. His is the first residue of a $\beta$-strand that follows a loop; thus, it is feasible that mobility of the loop enables the His to sample various conformations in the substrate-free form of CYLD. In both the Met1- and Lys63-linked diubiquitin complexes, the CYLD catalytic His is stabilized by hydrogen-bonding with Gln 2 of the proximal ubiquitin, promoting the active conformation.

Intriguingly, an added layer of protection from unwanted cleavage is seen in the pre-catalytic binding state of CYLD with Met1-linked diubiquitin [27]. The scissile peptide bond of the dimer was offset from the active site Cys by $8 \AA$, and a nearby loop that had been disordered in the unliganded CYLD structure was stabilized in the Met1- (and Lys63-) linked diubiquitin complex. Most interestingly, a Glu side chain in the loop apparently lies sandwiched between the scissile bond and the nucleophilic Cys, preventing catalytic activation (Figure 4C). Mutation of this residue to Gln led to its displacement from the active site, permitting proper orientation of the scissile peptide bond.
Substrate-induced rearrangement of catalytic residues into productive positions has also been observed in several SENP SUMO protease family members [35, 74-76]. Misaligned active site residues have yet to be observed in Nedd8- or ISG15-cleaving enzymes, but few structures have been examined to date. Examples of such misalignment may well be found in all types of DUBs and ULPs.

\section{Rearrangement of active site residues prevents oxidation} of catalytic cysteines

One rationale for why DUBs and ULPs might have evolved to adopt misaligned active sites in their substrate-free forms would be to prevent spurious activity against cellular proteins, while ensuring specificity toward the correct ubiquitin- or Ubl-linked conjugates [18]. Misalignment of catalytic residues may also limit oxidation of active-site cysteines [77-79]. Reactive oxygen species accumulate in cells in response to various types of stress, including UV, heat, and low levels of NADPH and glutathione [80]. Recently, proteases from the DUB and SENP families were found to be highly susceptible to oxidation of their catalytic cysteines, leading to accumulation of ubiquitin or SUMO conjugates in vivo [77, 79, 81, 82]. The Atg4 protease, which cleaves the Atg8 Ubl from phosphatidylethanolamine during autophagy, is also sensitive to oxidization [83].

Modification of a cysteine residue to sulfenic acid $(-\mathrm{SOH})$ can be reversed in the presence of reducing agents while formation of Cys-sulfinic acid $\left(-\mathrm{SO}_{2}\right)$ and Cys-sulfonic acid $\left(-\mathrm{SO}_{3}\right)$ is irreversible. The catalytic cysteines in SENPs can form intermolecular disulfide bonds, which would protect the Cys sulfhydryl from irreversible oxidation [79]. A20, a tumor suppressor that has both E3 ligase and OTU DUB domains, may use a distinct mechanism to protect the OTU active-site sulfhydryl. A crystal structure of A20 with its catalytic cysteine modified to sulfenic acid revealed that $\mathrm{Cys}-\mathrm{SOH}$ may limit further oxidation by engaging in several hydrogen bonds with a nearby loop [77].

The sulfhydryl group of most cysteine residues is less prone to oxidation since it maintains a $\mathrm{pKa}$ of $\sim 8.5$. However, in a catalytically competent active site, nucleophilic cysteines are more susceptible to oxidation because the His residue, acting as a general base, can deprotonate the Cys. The resulting thiolate anion can be readily oxidized (or can attack the scissile bond of a substrate). Thus, it seems plausible that by requiring realignment of active site residues in the presence of substrate, the reactive thiolate anion only forms when it can be used productively to catalyze cleavage of ubiquitin- or Ubl-linked conjugates. 
Active site loops that restrict substrates based on size

An active-site crossover loop is a key structural element unique to the UCH family of DUBs and has been proposed to play a critical role in selection of substrates [68]. This loop joins an $\alpha$-helix and $\beta$-sheet on opposite sides of the catalytic groove and ranges from 11 to 21 residues in length [84]. The UCHL1 crossover loop, the shortest known, assumes a somewhat rigid and open structure in the absence of substrate [60] that can accommodate the C-terminus of a ubiquitinated substrate [65]. In contrast, crystal structures of apo UCHL3 (Figure
5A) and UCHL5 (UCH37) reveal that the loop is largely disordered, suggesting that it can sample a variety of conformations in solution $[62,64,85,86]$. Binding of ubiquitin leads to apparent stabilization of the crossover loop, as it was seen straddling the active site in co-crystal structures of ubiquitin aldehyde bound to UCHL3 (Figure $5 \mathrm{~A}$ and 5B) and the yeast UCH Yuh1 $[62,87]$. The orientation of the ubiquitin C-terminal tail beneath the crossover loop indicates that a ubiquitinated substrate must be threaded through the loop, but the loop is too small to accommodate large substrates, including a ubiquitin dimer [60, 87]. Accordingly, UCH enzymes on their own are largely incapable of disassembling ubiquitin dimers
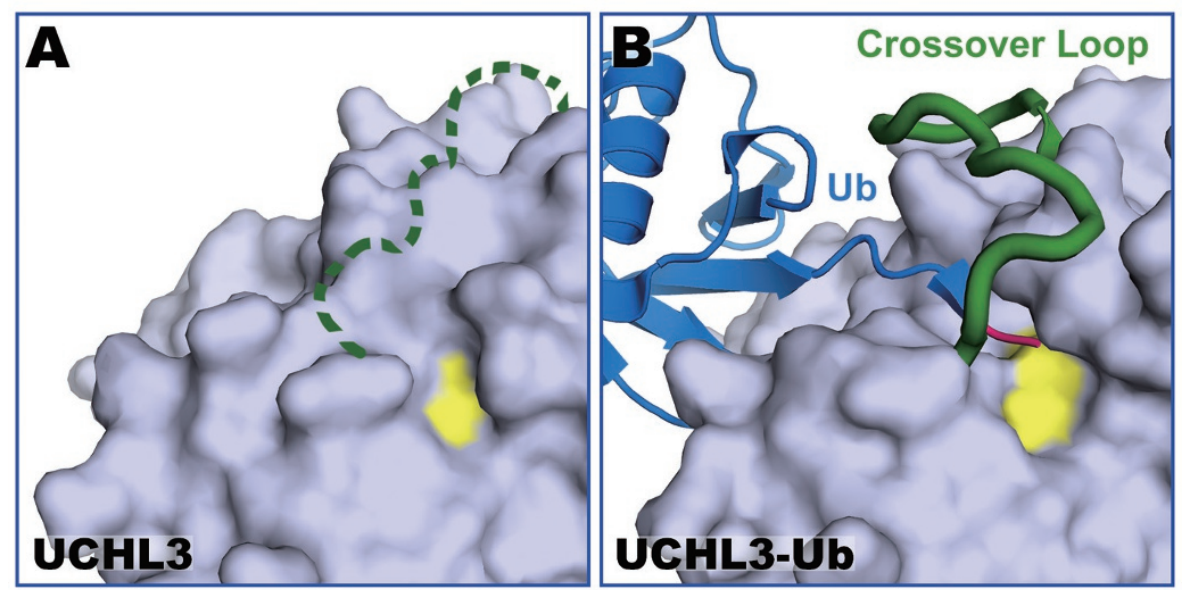

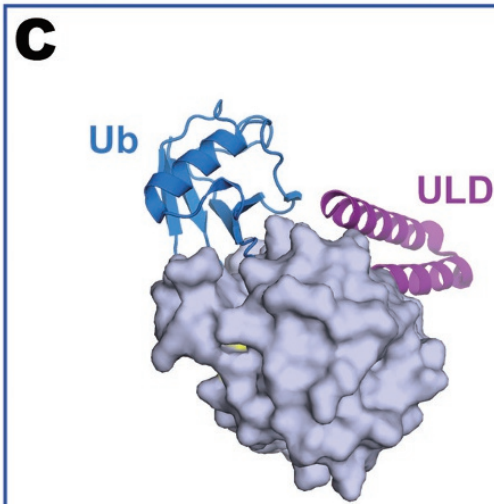

UCHL5-Ub

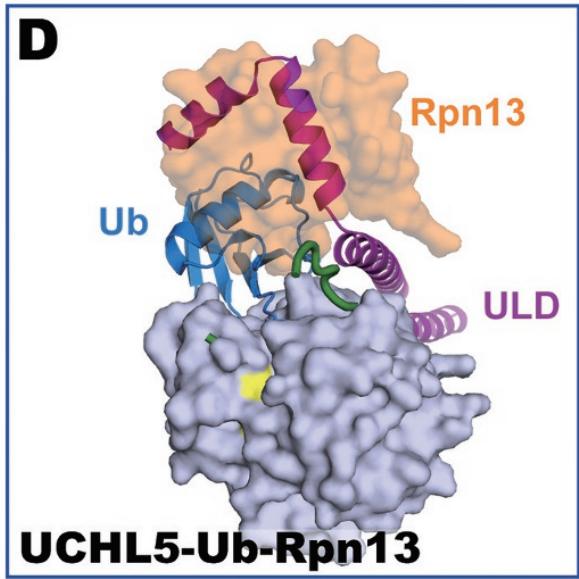

UCHL5-Ub-Rpn13

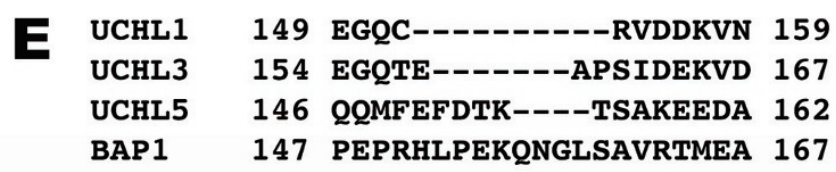

Figure $\mathbf{5}$ The active site crossover loop of $\mathrm{UCH}$ family enzymes is dynamic and restricts substrate size. In its substrate-free form, the crossover loop for UCHL3 is unstructured (A), but becomes ordered (green) when ubiquitin (colored blue) binds in the active site (B) (PDB codes: 1UCH [150], 1XD3 [87]). Surprisingly, even in the presence of ubiquitin, the active site crossover loop of UCHL5 remained disordered (PDB code: 4 IG7 [32]) (C). Binding of its cofactor, Rpn13, to UCHL5's C-terminal UCH37-like domain (ULD colored purple) (D), stabilizes a portion of the crossover loop (green; PDB code: 4WLR [67]). (E) Sequence alignment of the crossover loops indicates very little conservation. 
in vitro [55]. However, association of cellular cofactors, such as the proteasome ubiquitin receptor Rpn13 with UCHL5 or ASXL1 with BAP1, stimulates isopeptidase activity $[88,89]$.

Due to the steric constraints imposed by these crossover loops, especially in UCHL1 and UCHL3, which possess the shortest loops, it has been proposed that most substrates for this family of DUBs will have small leaving groups, including short precursor peptides, at the C-terminus of ubiquitin $[90,91]$. Indeed, extending or shortening the crossover loop can alter UCH specificity; at the same time, these loops are poorly conserved among $\mathrm{UCH}$ family members (Figure 5E). These results suggest that the size of the crossover loop, and not its sequence, is usually its key contribution to substrate selectivity $[84,91]$.

Surprisingly, even in the presence of ubiquitin, the crossover loop was found to be entirely unstructured in a UCHL5 ortholog from Trichinella spiralis (Figure 5C) [32]. Based on this observation, it was proposed that UCHL5 might require its proteasomal cofactor, Rpn13, to fully stabilize the loop. In fact, crystal structures of a ternary complex of UCHL5 bound to Rpn13 and ubiquitin $[66,67]$ revealed that a segment of the crossover loop (Met148 and Phe149) interacts directly with Rpn13, while the rest remains disordered (Figure 5D). These interactions pull a portion of the loop away from the active site, presumably opening it up for optimal binding of substrates. Rpn13 also binds to the C-terminal UCH37like domain (ULD) domain of the $\mathrm{UCH}$, locking the ULD into a favorable conformation for ubiquitin binding. A recent study suggests that ASXL1 might activate Bap1 in a similar manner [92].

\section{Insertions in catalytic domains contribute to sub- strate specificity}

The DUB and ULP enzyme families are defined by conserved sequence features of their catalytic domains. However, many bear unique terminal extensions or catalytic domain insertions. Here we will discuss several examples of how these insertions can contribute to the substrate specificity of individual enzymes in a family.

\section{Insertions in JAMM metalloproteases}

The JAMM family of metalloproteases share a conserved MPN core consisting of an 8 -stranded $\beta$-sheet sandwiched between two $\alpha$-helices, resembling a partially curled $\beta$ barrel $[93,94]$. Although sequence conservation is poor among family members, superposition of their MPN domains points toward conservation of the structural core, with small differences in the length of secondary structure elements [95]. Typically, JAMM proteins are found as part of complexes of at least two proteins, in which one subunit has an $\mathrm{MPN}^{+}$domain (the $\mathrm{MPN}^{+}$domain bears all the residues required for active site $\mathrm{Zn}^{2+}$ coordination - the "JAMM motif") and the other has an inactive MPN domain (JAMM motif absent). A key to substrate specificity in this class of enzymes originates from two divergent insertion segments, Ins-1 and Ins-2, which are unique to each family member (Figure 6A) [94, 96-101].

AMSH is a JAMM protease involved in endosomal sorting of ubiquitinated cell surface receptors [102]. Crystal structures of AMSH-like protein (AMSH-LP) and an AMSH ortholog from S. pombe bound to Lys63linked diubiquitin revealed that the molecular basis for AMSH's exquisite specificity for Lys63-linked chains arises largely from the Ins-2 segment and its binding to the proximal ubiquitin moiety $[33,103]$.

The C-terminal tail of the distal ubiquitin moiety binds the active site cleft in an extended $\beta$-strand conformation and is stabilized by extensive hydrogen bonding with a $\beta$-strand from the Ins- 1 segment. These interactions between distal ubiquitin and Ins-1 position the scissile isopeptide bond for hydrolysis. The Ins-2 segment of AMSH is a loop that forms a flap structure near the active site and is stabilized by coordination of a second, non-catalytic $\mathrm{Zn}^{2+}$ ion. Ins-2 dictates substrate specificity for Lys63-linked ubiquitin chains by hydrogen bonding with Gln62 and Glu64 of the proximal ubiquitin moiety while a conserved Phe from the flap region appears to clamp down on the isopeptide bond of diubiquitin [33, 103]. Mutation of the conserved Phe led to reduced selectivity for Lys63-linked diubiquitin, possibly due to increased flexibility of the Ins-2 loop [104]. Binding to its cofactor STAM2 stimulates AMSH deubiquitinating activity. A UIM-SH3 domain from STAM2 binds to both AMSH and the Ile44 patch on the proximal ubiquitin, serving to further stabilize the position of the proximal ubiquitin moiety on the DUB $[102,105,106]$.

CSN5 is a JAMM protease that is a part of a protein complex known as the COP9 signalosome (CSN) [107]. The main role of CSN5 is to deneddylate Cullin-Ring E3 ligases (CRLs) [108, 109]; however, the activity of CSN5 is severely reduced when it is not part of the CSN [110, 111]. The Ins-1 region of CSN5 was recently shown to occlude the active site, thus preventing Nedd8 binding, and its high crystallographic B factors point to a loop structure that can assume multiple conformations [96]. The crystal structure of CSN showed that like Rpn11 (see below), CSN5 is seemingly auto-inhibited by altering tetrahedral coordination of the active site $\mathrm{Zn}^{2+}$ ion [99]. Glu104 of the Ins-1 segment occupies the fourth coor- 

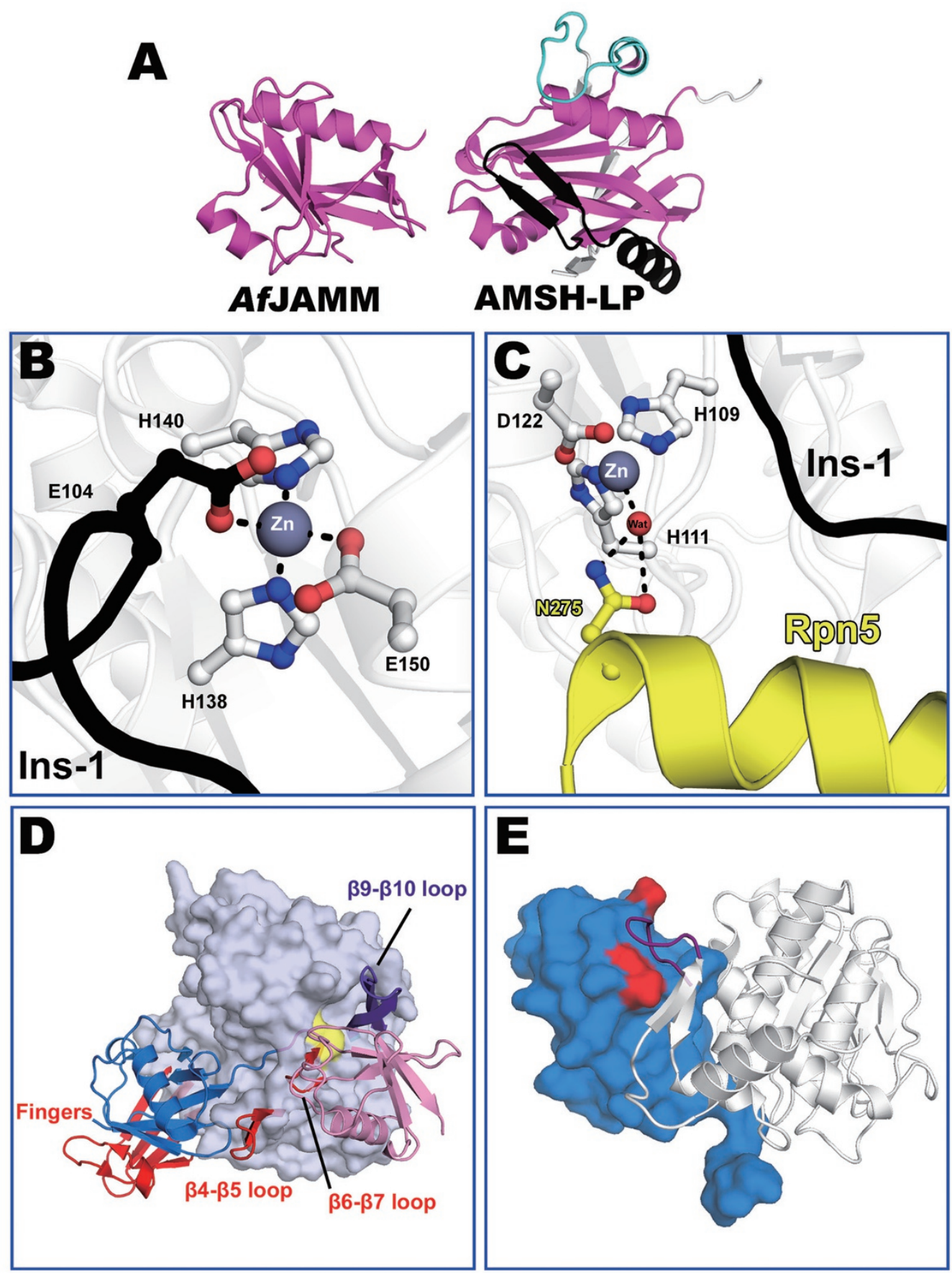

Figure 6 Insertions within the catalytic domain of ULPs also contribute to substrate specificity. (A) Ins-1 (black) and Ins-2 (cyan) segments of AMSH-LP are shown within the JAMM core of AMSH-LP (PDB code: 2ZNR [33]), compared to the protease AfJAMM (PDB code: 1R5X [93]). (B) E104 of the Ins-1 segment of CSN5, colored black, stabilizes the Ins-1 loop in an inactive conformation (PDB code: 4D10 [99]). (C) N275 of a nearby proteasome lid subunit, Rpn5 (yellow), appears to inhibit Rpn11 by serving as the fourth ligand in a water-mediated interaction with the Rpn11 active site zinc (PDB code: 3JCK [116]). This locks the Ins-1 segment of Rpn11 in a closed conformation. (D) CYLD specificity for Met1- and Lys63-linked polyubiquitin is linked to truncations of various structural elements within the catalytic domain compared to other USP family members (PDB codes: 3WXF [27], 1NB8 [28]). Shown in red are structural features of USP7 that are absent in CYLD. These truncations and an insertion unique to CYLD (purple) shift polyubiquitin recognition from the distal ubiquitin (blue) to the proximal ubiquitin (pink). (E) A chimeric construct of SENP2 containing the Loop-1 insertion of SENP6 (purple; PDB code: $3 Z$ O5 [120]) illustrates SENP6/7 preference for SUMO2/3 (SUMO2 is colored blue), as the loop binds to a negative patch of residues (red) not conserved in SUMO1. 
dination spot of $\mathrm{Zn}^{2+}$, dispelling the nucleophilic water from the active site and stabilizing CSN5 in an inactive conformation (Figure 6B). Thus, activation of CSN5 deneddylase activity likely requires a major conformational change of the Ins-1 segment, which is probably induced by binding of a neddylated CRL [99].

\section{The proteasomal DUB Rpn 11}

Another JAMM family DUB, Rpn11, is one of nine subunits of the proteasome 'lid', a subcomplex of the regulatory particle (RP) of the proteasome [112]. Substrates that have been earmarked for degradation by the proteasome are deubiquitinated en bloc by Rpn11 in an ATP-dependent manner $[89,94]$. Rpn11 is most active as part of the full proteasome; it is inactive when purified on its own or in the isolated lid subcomplex [113]. Rpn8, which possesses an inactive MPN domain, forms a heterodimer with Rpn11, and this complex displays residual DUB activity in vitro [114]. Recent crystal structures of the Rpn8-Rpn11 heterodimer revealed that the Ins-2 segment of Rpn11 has a function entirely different from that of the Ins-2 loop of AMSH $[115,116]$. In the Rpn8Rpn11 structures, the Ins-2 loop was disordered; however, modeling the crystal structure into the averaged electron density used for a proteasome cryoEM reconstruction indicated that the Ins-2 loop interacts with Rpn2, a subunit belonging to the subcomplex of the RP known as the 'base', to aid in the positioning of Rpn11. The ability of Rpn11 to cleave all seven polyubiqiuitin lysine linkages may arise from the absence of structural elements that contact the proximal ubiquitin [116].

The structural studies also showed that Rpn11 Ins-1 differs vastly in structure from Ins-1 of AMSH-LP and appears to occlude the Rpn11 active site. However, instead of augmenting DUB activity, mutations in the Ins-1 loop impair it, suggesting that the loop is not inhibitory, but is in fact necessary for catalysis. High crystallographic B-factors suggest that in the absence of substrates, the Ins-1 loop is dynamic. It was proposed that once an ubiquitinated substrate enters the catalytic site, Ins-1 will clamp down over the substrate to help position it for cleavage [116].

A recent $3.5 \AA$ cryoEM structure of the proteasome lid offers further insight into what keeps Rpn11 inactive when outside of the full proteasome [117]. An $\alpha$-helix of the lid subunit Rpn5 sterically blocks the top of the Rpn11 catalytic cleft, and several residues from the $\mathrm{N}$-terminal end of the $\alpha$-helix directly interact with loops surrounding the catalytic $\mathrm{Zn}^{2+}$ ion. Strikingly, Asn275 from Rpn5 appears to insert itself near the Rpn11 active site and stabilize tetrahedral coordination of its $\mathrm{Zn}^{2+}$ through a bridging interaction with a coordinated water molecule (Figure 6C). These interactions may lock the Ins-1 loop in a closed conformation, occluding the active site. When the lid is incorporated into the proteasome, conformational changes in the Rpn11-Rpn8 heterodimer are proposed to lead to its activation through distortion of the Rpn5-Rpn11 and Rpn9-Rpn8 contact sites.

\section{Insertions in the catalytic domains of USP DUBs and SENP ULPS}

Like JAMM domains, USP catalytic domains, which range in size from $\sim 300$ to $\sim 900$ residues, also often have insertions. A detailed analysis of USP domain architecture revealed that within the conserved USP core there are five potential loop locations for insertions [118]. In most cases, the insertions are predicted to fold into independent domains, and depending on the location, may influence DUB activity [118]. A striking example of how an insertion in the catalytic domain affects substrate specificity comes again from CYLD, a USP that deviates from the canonical USP fold due to several truncations and an insertion [27, 73]. As mentioned earlier, CYLD is highly specific for Met1 and Lys63 ubiquitin linkages. Truncation of structural elements typically involved in distal ubiquitin binding in other USPs reduces the affinity of CYLD for ubiquitin (Figure 6D). At the same time, a unique insertion segment for CYLD, the $\beta 9-\beta 10$ strands, interacts with the Phe4 hydrophobic patch of the proximal ubiquitin (Figure 6D), an interaction specific to Met1 and Lys63 linkages [27]. Deletion of the $\beta 9-\beta 10$ segment diminishes DUB activity against Lys63 ubiquitin linkages, underscoring its role in substrate specificity [73]. Furthermore, Glu16 of the proximal ubiquitin moiety occupies a CYLD binding pocket created as a result of truncation of the $\beta 6-\beta 7$ loop. These modifications to the CYLD USP domain architecture contribute to specificity by shifting the burden of ubiquitin recognition from the distal ubiquitin to the proximal ubiquitin molecule.

The mammalian SUMO proteases SENP6 and SENP7 display high specificity for the SUMO2/3 isoforms (which are nearly identical) over the SUMO1 protein. They are characterized by the presence of four loop insertions within their (poorly conserved) catalytic domains. The crystal structure of SENP7 revealed that the Loop-1 and Loop-2 insertions are found on the protease surface at positions that would likely contact SUMO [119]. Deletion of Loop-1, but not Loop-2, impaired SENP7 SUMO2/3-cleaving activity, suggesting that Loop-1 plays a key role in determining the specificity of SENP7 for SUMO2/3 [119]. The crystal structure of a chimeric SENP2 fusion harboring the Loop-1 segment from SENP6 bound to SUMO2 revealed that Loop-1, an eight-residue element, extends the binding interface 
between the protease and SUMO2. A negative patch of amino acids unique to SUMO2 (Asn68, Asp71 and Glu77) directly contacts the SENP6 Loop-1 (Figure 6E) [120]. These residues are substituted to Ala, His and Gly in SUMO1, which implicates both the Loop-1 insertion in SENP6/7 and the negative patch of SUMO2 as key determinants in SUMO isoform specificity.

\section{Substrate specificity of non-eukaryotic DUBs and ULPs}

Although ubiquitin/Ubl modification systems were originally thought to be unique to eukaryotes, antecedents of all components of these systems have now been detected in archaea and bacteria [14, 121]. Among the proteases that (putatively) cleave Ubls from prokaryotic proteins, the JAMM proteases are the most common based on bioinformatic analyses [121]. Viruses also encode select components of these systems [122]. Interestingly, a number of DUBs and ULPs have been identified in pathogenic bacteria and bacteria that reside within eukaryotic cells [123]. These enzymes are often most similar in sequence to eukaryotic enzymes, suggesting possible horizontal gene transfer [26, 124, 125], although in some cases their ability to cleave ubiquitin or Ubls may have evolved independently from a primordial protease core [126]. No Ubl-type modifier systems exist in these bacteria; instead these ubiquitin- or Ubl-specific proteases are injected as effector proteins through a specialized secretion apparatus into the eukaryotic host [127-129].

ULPs and DUBs have been described in the genomes of obligate intracellular and pathogenic gram-negative strains of bacteria. These enzymes include the ChlaDUBs and ChlaOTU of Chlamydia [128, 130, 131], the putative $w$ Pa_0283 ULP of Wolbachia [124], a putative USP from Cardinium [132], SdeA of Legionella [26, 133], SseL of Salmonella [134], XopD of Xanthomonas [129, 135], and ElaD of E. coli [125]. Certain viruses also encode ULPs or DUBs. Examples include DNA viruses of the Adenoviridae, Poxviridae and Herpesviridae families $[122,136]$, and RNA viruses such as Crimean Congo Hemorrhagic Fever Virus (CCHFV) and Turnip Yellow Mosaic Virus (TYMV), which have OTU proteases [137].

These bacterial and viral proteases have provided useful models for predicting the ubiquitin and Ubl specificity of DUBs and ULPs. Surprisingly, many turn out to have specificities different from the eukaryotic enzymes to which they are most closely related. For example, the C48 Ulp1-like family of enzymes that includes yeast Ulp1, ElaD, and SdeA are all expected to share similar folds and catalytic sites and thus might have been expected to cleave SUMO conjugates based on the original studies on Ulp1 [126]. However, these enzymes have very different Ubl preferences: Ulp1 cleaves SUMO [126, 138]; ElaD cleaves ubiquitin [125]; and SdeA cleaves both ubiquitin and Nedd8 [26].

Viral ULPs generally cleave both viral precursor proteins and host Ubl-protein conjugates. A well-studied example is the adenovirus proteinase (AVP) which is a member of the C48 Ulp1-like protease family [139]. AVP cleaves specific virion precursor proteins to their mature forms [140, 141]. Interestingly, AVP was identified in a screen for DUBs using an ubiquitin-aldehyde inhibitor and found to have DUB activity against both ubiquitin and ISG15 [122]. Despite its closest eukaryotic counterparts being the SUMO-cleaving ULPs, AVP fails to cleave SUMO substrates. Its inability to cleave SUMO can be explained by the lack of specific sequence elements upstream of the core Ulp1 protease domain that contact SUMO [74, 126]; however, its dual specificity for ubiquitin and ISG15 has not yet been rationalized [122].

Viral OTU proteases, such as the enzyme from $\mathrm{CCH}-$ FV, also cleave both ubiquitin and ISG15 conjugates [142]. This broadened specificity compared to eukaryotic OTU enzymes, which are ubiquitin-specific, was traced to the manner by which the CCHFV OTU protease positions its substrates. From crystallographic data, the bound ubiquitin and C-terminal Ubl domain of ISG15 were rotated $75^{\circ}$ on the viral protease surface relative to ubiquitin bound to a yeast OTU protease. Different viral enzyme determinants direct ISG15 and ubiquitin binding, allowing its specificity to be manipulated by specific mutations [143, 144]. A similar rotation was observed for substrate binding to an arterivirus OTU [145]. These results show that structural data from DUB/ULP-substrate complexes permit ubiquitin and Ubl specificity to be dissected experimentally.

An interesting example of the challenges in predicting Ubl protease substrate specificity comes from a study of XopD, a C48 Ulp1-like protease derived from a plant pathogen [138]. XopD is the only verified prokaryotic SUMO protease. The XopD crystal structure showed a similar fold to yeast Ulp1 from a Ulp1-SUMO co-crystal structure [74], but the two enzymes have distinct specificities. C-terminal residues in particular plant SUMOs upstream of the cleavage site appear to guide XopD binding to these specific SUMO isoforms. By contrast, Ulp1 is far more promiscuous and can cleave not only these plant SUMOs, but also yeast and mammalian SUMO-fusion substrates. Among other differences, a conserved hydrophobic residue in Ulp1, Phe474, which is required for its function, has been replaced in XopD orthologs by glutamine. Similarly, this residue is altered in the Ulp1like ChlaDUBs, but these enzymes hydrolyze ubiquitin 
rather than SUMO; it is also a glutamine in SENP8/ Den1, which is a deneddylase [138]. Therefore, the Phe474-equivalent position in Ulp1-like enzymes may be important for dictating Ubl preferences, but it remains difficult to predict what those preferences are.

\section{Concluding remarks}

Although many advances have been made over the past $\sim 25$ years in understanding the molecular basis of substrate specificity of DUBs and ULPs, many questions linger. An issue we sought to address at the outset of this review concerned whether Ubl or ubiquitin (chain) specificity for a particular DUB or ULP could be inferred solely from its amino acid sequence. This remains very challenging due, as outlined here, to the multiple mechanisms used for discrimination among substrates. Low sequence conservation among DUBs and ULPs adds to the difficulty. Further structural and biochemical studies of ubiquitin/Ubl proteases and their substrates should enhance our ability to predict the function and specificity of these proteases and to understand their detailed mechanisms.

\section{Acknowledgments}

We thank Chittaranjan Das and Christopher Hickey for their helpful comments on the manuscript. Work from our lab was supported by USDA (2014-67012-22268) to JFB and US NIH grants (GM046904, GM053756 and GM083050) to MH.

\section{References}

1 Glickman MH, Ciechanover A. The ubiquitin-proteasome proteolytic pathway: destruction for the sake of construction. Physiol Rev 2002; 82:373-428.

2 Hershko A, Ciechanover A. The ubiquitin system. Annu Rev Biochem 1998; 67:425-479.

3 Komander D, Rape M. The ubiquitin code. Annu Rev Biochem 2012; 81:203-229.

4 Varshavsky A. The ubiquitin system. Trends Biochem Sci 1997; 22:383-387.

5 Pickart CM, Eddins MJ. Ubiquitin: structures, functions, mechanisms. Biochim Biophys Acta 2004; 1695:55-72.

6 Schulman BA, Harper JW. Ubiquitin-like protein activation by E1 enzymes: the apex for downstream signalling pathways. Nat Rev Mol Cell Biol 2009; 10:319-331.

7 Haglund K, Dikic I. Ubiquitylation and cell signaling. EMBO J 2005; 24:3353-3359.

8 Ikeda F, Dikic I. Atypical ubiquitin chains: new molecular signals. 'Protein Modifications: Beyond the Usual Suspects' review series. EMBO Rep 2008; 9:536-542.

9 Pickart CM. Ubiquitin in chains. Trends Biochem Sci 2000; 25:544-548.

10 Ben-Saadon R, Zaaroor D, Ziv T, Ciechanover A. The poly- comb protein Ring1B generates self atypical mixed ubiquitin chains required for its in vitro histone H2A ligase activity. Mol Cell 2006; 24:701-711.

11 Boname JM, Thomas M, Stagg HR, Xu P, Peng J, Lehner PJ. Efficient internalization of MHC I requires lysine-11 and lysine-63 mixed linkage polyubiquitin chains. Traffic 2010; 11:210-220.

12 Kravtsova-Ivantsiv Y, Ciechanover A. Non-canonical ubiquitin-based signals for proteasomal degradation. J Cell Sci 2012; 125:539-548.

13 Newton K, Matsumoto ML, Wertz IE, et al. Ubiquitin chain editing revealed by polyubiquitin linkage-specific antibodies. Cell 2008; 134:668-678.

14 Hochstrasser M. Origin and function of ubiquitin-like proteins. Nature 2009; 458:422-429.

15 Schulman BA. Twists and turns in ubiquitin-like protein conjugation cascades. Protein Sci 2011; 20:1941-1954.

16 Hickey CM, Wilson NR, Hochstrasser M. Function and regulation of SUMO proteases. Nat Rev Mol Cell Biol 2012; 13:755-766.

17 Guzzo CM, Matunis MJ. Expanding SUMO and ubiquitin-mediated signaling through hybrid SUMO-ubiquitin chains and their receptors. Cell Cycle 2013; 12:1015-1017.

18 Amerik AY, Hochstrasser M. Mechanism and function of deubiquitinating enzymes. Biochim Biophys Acta 2004; 1695:189-207.

19 Komander D. Mechanism, specificity and structure of the deubiquitinases. Subcell Biochem 2010; 54:69-87.

20 Komander D, Clague MJ, Urbe S. Breaking the chains: structure and function of the deubiquitinases. Nat Rev Mol Cell Biol 2009; 10:550-563.

21 Nijman SM, Luna-Vargas MP, Velds A, et al. A genomic and functional inventory of deubiquitinating enzymes. Cell 2005; 123:773-786.

22 Beal R, Deveraux Q, Xia G, Rechsteiner M, Pickart C. Surface hydrophobic residues of multiubiquitin chains essential for proteolytic targeting. Proc Natl Acad Sci USA 1996; 93:861-866.

23 Sloper-Mould KE, Jemc JC, Pickart CM, Hicke L. Distinct functional surface regions on ubiquitin. J Biol Chem 2001; 276:30483-30489.

24 Reverter D, Wu K, Erdene TG, Pan ZQ, Wilkinson KD, Lima CD. Structure of a complex between Nedd8 and the Ulp/Senp protease family member Den1. J Mol Biol 2005; 345:141151.

25 Shen LN, Liu H, Dong C, Xirodimas D, Naismith JH, Hay RT. Structural basis of NEDD8 ubiquitin discrimination by the deNEDDylating enzyme NEDP1. EMBO J 2005; 24:1341-1351.

26 Sheedlo MJ, Qiu J, Tan Y, Paul LN, Luo ZQ, Das C. Structural basis of substrate recognition by a bacterial deubiquitinase important for dynamics of phagosome ubiquitination. Proc Natl Acad Sci USA 2015; 112:15090-15095.

27 Sato Y, Goto E, Shibata Y, et al. Structures of CYLD USP with Met1- or Lys63-linked diubiquitin reveal mechanisms for dual specificity. Nat Struct Mol Biol 2015; 22:222-229.

$28 \mathrm{Hu} \mathrm{M}$, Li P, Li M, et al. Crystal structure of a UBP-family deubiquitinating enzyme in isolation and in complex with ubiquitin aldehyde. Cell 2002; 111:1041-1054. 
29 Lange OF, Lakomek NA, Fares C, et al. Recognition dynamics up to microseconds revealed from an RDC-derived ubiquitin ensemble in solution. Science 2008; 320:1471-1475.

30 Ye Y, Akutsu M, Reyes-Turcu F, Enchev RI, Wilkinson KD, Komander D. Polyubiquitin binding and cross-reactivity in the USP domain deubiquitinase USP21. EMBO Rep 2011; 12:350-357.

31 Vijay-Kumar S, Bugg CE, Cook WJ. Structure of ubiquitin refined at 1.8 Å resolution. J Mol Biol 1987; 194:531-544.

32 Morrow ME, Kim MI, Ronau JA, et al. Stabilization of an unusual salt bridge in ubiquitin by the extra C-terminal domain of the proteasome-associated deubiquitinase UCH37 as a mechanism of its exo specificity. Biochemistry 2013; 52:35643578.

33 Sato Y, Yoshikawa A, Yamagata A, et al. Structural basis for specific cleavage of Lys 63-linked polyubiquitin chains. $\mathrm{Na}$ ture 2008; 455:358-362.

34 Cappadocia L, Pichler A, Lima CD. Structural basis for catalytic activation by the human ZNF451 SUMO E3 ligase. Nat Struct Mol Biol 2015; 22:968-975.

35 Reverter D, Lima CD. A basis for SUMO protease specificity provided by analysis of human Senp2 and a Senp2-SUMO complex. Structure 2004; 12:1519-1531.

36 Reverter D, Lima CD. Structural basis for SENP2 protease interactions with SUMO precursors and conjugated substrates. Nat Struct Mol Biol 2006; 13:1060-1068.

37 Phillips AH, Zhang Y, Cunningham CN, et al. Conformational dynamics control ubiquitin-deubiquitinase interactions and influence in vivo signaling. Proc Natl Acad Sci USA 2013; 110:11379-11384.

38 Kazlauskaite A, Kondapalli C, Gourlay R, et al. Parkin is activated by PINK1-dependent phosphorylation of ubiquitin at Ser65. Biochem J 2014; 460:127-139.

39 Koyano F, Okatsu K, Kosako H, et al. Ubiquitin is phosphorylated by PINK1 to activate parkin. Nature 2014; 510:162166.

40 Swaney DL, Beltrao P, Starita L, et al. Global analysis of phosphorylation and ubiquitylation cross-talk in protein degradation. Nat Methods 2013; 10:676-682.

41 Wauer T, Swatek KN, Wagstaff JL, et al. Ubiquitin Ser65 phosphorylation affects ubiquitin structure, chain assembly and hydrolysis. EMBO J 2015; 34:307-325.

42 Swaney DL, Rodriguez-Mias RA, Villen J. Phosphorylation of ubiquitin at Ser65 affects its polymerization, targets, and proteome-wide turnover. EMBO Rep 2015; 16:1131-1144.

43 Datta AB, Hura GL, Wolberger C. The structure and conformation of Lys63-linked tetraubiquitin. J Mol Biol 2009; 392:1117-1124.

44 Weeks SD, Grasty KC, Hernandez-Cuebas L, Loll PJ. Crystal structures of Lys-63-linked tri- and di-ubiquitin reveal a highly extended chain architecture. Proteins 2009; 77:753-759.

45 Komander D, Reyes-Turcu F, Licchesi JD, Odenwaelder $\mathrm{P}$, Wilkinson KD, Barford D. Molecular discrimination of structurally equivalent Lys 63-linked and linear polyubiquitin chains. EMBO Rep 2009; 10:466-473.

46 Cook WJ, Jeffrey LC, Carson M, Chen Z, Pickart CM. Structure of a diubiquitin conjugate and a model for interaction with ubiquitin conjugating enzyme (E2). J Biol Chem 1992; 267:16467-16471.
47 Eddins MJ, Varadan R, Fushman D, Pickart CM, Wolberger C. Crystal structure and solution NMR studies of Lys48-linked tetraubiquitin at neutral pH. J Mol Biol 2007; 367:204-211.

48 Bremm A, Freund SM, Komander D. Lys11-linked ubiquitin chains adopt compact conformations and are preferentially hydrolyzed by the deubiquitinase Cezanne. Nat Struct Mol Biol 2010; 17:939-947.

49 Hospenthal MK, Freund SM, Komander D. Assembly, analysis and architecture of atypical ubiquitin chains. Nat Struct Mol Biol 2013; 20:555-565.

50 Matsumoto ML, Wickliffe KE, Dong KC, et al. K11-linked polyubiquitination in cell cycle control revealed by a $\mathrm{K} 11$ linkage-specific antibody. Mol Cell 2010; 39:477-484.

51 Kristariyanto YA, Choi SY, Rehman SA, et al. Assembly and structure of Lys33-linked polyubiquitin reveals distinct conformations. Biochem J 2015; 467:345-352.

52 Hirano T, Serve O, Yagi-Utsumi M, et al. Conformational dynamics of wild-type Lys-48-linked diubiquitin in solution. $J$ Biol Chem 2011; 286:37496-37502.

53 Rohaim A, Kawasaki M, Kato R, Dikic I, Wakatsuki S. Structure of a compact conformation of linear diubiquitin. Acta Crystallogr D Biol Crystallogr 2012; 68:102-108.

54 Cooper EM, Cutcliffe C, Kristiansen TZ, Pandey A, Pickart CM, Cohen RE. K63-specific deubiquitination by two JAMM/ MPN+ complexes: BRISC-associated Brcc36 and proteasomal Poh1. EMBO J 2009; 28:621-631.

55 Ritorto MS, Ewan R, Perez-Oliva AB, et al. Screening of DUB activity and specificity by MALDI-TOF mass spectrometry. Nat Commun 2014; 5:4763.

56 Davies CW, Paul LN, Kim MI, Das C. Structural and thermodynamic comparison of the catalytic domain of AMSH and AMSH-LP: nearly identical fold but different stability. $J \mathrm{Mol}$ Biol 2011; 413:416-429.

57 Faesen AC, Luna-Vargas MP, Geurink PP, et al. The differential modulation of USP activity by internal regulatory domains, interactors and eight ubiquitin chain types. Chem Biol 2011; 18:1550-1561.

58 Mevissen TE, Hospenthal MK, Geurink PP, et al. OTU deubiquitinases reveal mechanisms of linkage specificity and enable ubiquitin chain restriction analysis. Cell 2013; 154:169184.

59 Messick TE, Russell NS, Iwata AJ, et al. Structural basis for ubiquitin recognition by the Otul ovarian tumor domain protein. J Biol Chem 2008; 283:11038-11049.

60 Das C, Hoang QQ, Kreinbring CA, et al. Structural basis for conformational plasticity of the Parkinson's disease-associated ubiquitin hydrolase UCH-L1. Proc Natl Acad Sci USA 2006; 103:4675-4680.

61 Edelmann MJ, Iphofer A, Akutsu M, et al. Structural basis and specificity of human otubain 1-mediated deubiquitination. Biochem J 2009; 418:379-390.

62 Johnston SC, Riddle SM, Cohen RE, Hill CP. Structural basis for the specificity of ubiquitin $\mathrm{C}$-terminal hydrolases. $E M B O$ $J$ 1999; 18:3877-3887.

63 Keusekotten K, Elliott PR, Glockner L, et al. OTULIN antagonizes LUBAC signaling by specifically hydrolyzing Met1linked polyubiquitin. Cell 2013; 153:1312-1326.

64 Maiti TK, Permaul M, Boudreaux DA, Mahanic C, Mauney S, Das C. Crystal structure of the catalytic domain of UCHL5, 
a proteasome-associated human deubiquitinating enzyme, reveals an unproductive form of the enzyme. FEBS $J$ 2011; 278:4917-4926.

65 Boudreaux DA, Maiti TK, Davies CW, Das C. Ubiquitin vinyl methyl ester binding orients the misaligned active site of the ubiquitin hydrolase UCHL1 into productive conformation. Proc Natl Acad Sci USA 2010; 107:9117-9122.

66 Sahtoe DD, van Dijk WJ, El Oualid F, Ekkebus R, Ovaa H, Sixma TK. Mechanism of UCH-L5 activation and inhibition by DEUBAD domains in RPN13 and INO80G. Mol Cell 2015; 57:887-900.

67 VanderLinden RT, Hemmis CW, Schmitt B, et al. Structural basis for the activation and inhibition of the UCH37 deubiquitylase. Mol Cell 2015; 57:901-911.

68 Reyes-Turcu FE, Ventii KH, Wilkinson KD. Regulation and cellular roles of ubiquitin-specific deubiquitinating enzymes. Annu Rev Biochem 2009; 78:363-397.

69 Hibi K, Liu Q, Beaudry GA, et al. Serial analysis of gene expression in non-small cell lung cancer. Cancer Res 1998; 58:5690-5694.

70 Leroy E, Boyer R, Auburger G, et al. The ubiquitin pathway in Parkinson's disease. Nature 1998; 395:451-452.

71 Fiil BK, Damgaard RB, Wagner SA, et al. OTULIN restricts Met1-linked ubiquitination to control innate immune signaling. Mol Cell 2013; 50:818-830.

72 Rivkin E, Almeida SM, Ceccarelli DF, et al. The linear ubiquitin-specific deubiquitinase gumby regulates angiogenesis. Nature 2013; 498:318-324.

73 Komander D, Lord CJ, Scheel H, et al. The structure of the CYLD USP domain explains its specificity for Lys63-linked polyubiquitin and reveals a B box module. Mol Cell 2008; 29:451-464.

74 Mossessova E, Lima CD. Ulp1-SUMO crystal structure and genetic analysis reveal conserved interactions and a regulatory element essential for cell growth in yeast. Mol Cell 2000; 5:865-876.

75 Shen LN, Dong C, Liu H, Naismith JH, Hay RT. The structure of SENP1-SUMO-2 complex suggests a structural basis for discrimination between SUMO paralogues during processing. Biochem J 2006; 397:279-288.

$76 \mathrm{Xu}$ Z, Chau SF, Lam KH, Chan HY, Ng TB, Au SW. Crystal structure of the SENP1 mutant C603S-SUMO complex reveals the hydrolytic mechanism of SUMO-specific protease. Biochem J 2006; 398:345-352.

77 Kulathu Y, Komander D. Atypical ubiquitylation - the unexplored world of polyubiquitin beyond Lys48 and Lys63 linkages. Nat Rev Mol Cell Biol 2012; 13:508-523.

78 Wolberger C. Mechanisms for regulating deubiquitinating enzymes. Protein Sci 2014; 23:344-353.

79 Xu Z, Lam LS, Lam LH, Chau SF, Ng TB, Au SW. Molecular basis of the redox regulation of SUMO proteases: a protective mechanism of intermolecular disulfide linkage against irreversible sulfhydryl oxidation. FASEB J 2008; 22:127-137.

80 Holmstrom KM, Finkel T. Cellular mechanisms and physiological consequences of redox-dependent signalling. Nat Rev Mol Cell Biol 2014; 15:411-421.

81 Cotto-Rios XM, Bekes M, Chapman J, Ueberheide B, Huang TT. Deubiquitinases as a signaling target of oxidative stress. Cell Rep 2012; 2:1475-1484.
82 Lee JG, Baek K, Soetandyo N, Ye Y. Reversible inactivation of deubiquitinases by reactive oxygen species in vitro and in cells. Nat Commun 2013; 4:1568.

83 Scherz-Shouval R, Shvets E, Fass E, Shorer H, Gil L, Elazar Z. Reactive oxygen species are essential for autophagy and specifically regulate the activity of Atg4. EMBO J 2007; 26:1749-1760.

84 Zhou ZR, Zhang YH, Liu S, Song AX, Hu HY. Length of the active-site crossover loop defines the substrate specificity of ubiquitin C-terminal hydrolases for ubiquitin chains. Biochem J 2012; 441:143-149.

85 Artavanis-Tsakonas K, Weihofen WA, Antos JM, et al. Characterization and structural studies of the Plasmodium falciparum ubiquitin and Nedd8 hydrolase UCHL3. J Biol Chem 2010; 285:6857-6866.

86 Burgie SE, Bingman CA, Soni AB, Phillips GN Jr. Structural characterization of human Uch37. Proteins 2012; 80:649-654.

87 Misaghi S, Galardy PJ, Meester WJ, Ovaa H, Ploegh HL, Gaudet R. Structure of the ubiquitin hydrolase UCH-L3 complexed with a suicide substrate. J Biol Chem 2005; 280:15121520.

88 Scheuermann JC, de Ayala Alonso AG, Oktaba K, et al. Histone $\mathrm{H} 2 \mathrm{~A}$ deubiquitinase activity of the Polycomb repressive complex PR-DUB. Nature 2010; 465:243-247.

89 Yao T, Song L, Xu W, et al. Proteasome recruitment and activation of the Uch37 deubiquitinating enzyme by Adrm1. Nat Cell Biol 2006; 8:994-1002.

90 Larsen CN, Krantz BA, Wilkinson KD. Substrate specificity of deubiquitinating enzymes: ubiquitin C-terminal hydrolases. Biochemistry 1998; 37:3358-3368.

91 Popp MW, Artavanis-Tsakonas K, Ploegh HL. Substrate filtering by the active site crossover loop in UCHL3 revealed by sortagging and gain-of-function mutations. J Biol Chem 2009; 284:3593-3602.

92 Sahtoe DD, van Dijk WJ, Ekkebus R, Ovaa H, Sixma TK. BAP1/ASXL1 recruitment and activation for H2A deubiquitination. Nat Commun 2016; 7:10292.

93 Ambroggio XI, Rees DC, Deshaies RJ. JAMM: a metalloprotease-like zinc site in the proteasome and signalosome. PLoS Biol 2004; 2:E2.

94 Verma R, Aravind L, Oania R, et al. Role of Rpn11 metalloprotease in deubiquitination and degradation by the $26 \mathrm{~S}$ proteasome. Science 2002; 298:611-615.

95 Birol M, Echalier A. Structure and function of MPN (Mpr1/ Pad1 N-terminal) domain-containing proteins. Curr Protein Pept Sci 2014; 15:504-517.

96 Echalier A, Pan Y, Birol M, et al. Insights into the regulation of the human COP9 signalosome catalytic subunit, CSN5/ Jab1. Proc Natl Acad Sci USA 2013; 110:1273-1278.

97 Guterman A, Glickman MH. Deubiquitinating enzymes are IN/(trinsic to proteasome function). Curr Protein Pept Sci 2004; 5:201-211.

98 Kyuuma M, Kikuchi K, Kojima K, et al. AMSH, an ESCRT-III associated enzyme, deubiquitinates cargo on MVB/ late endosomes. Cell Struct Funct 2007; 31:159-172.

99 Lingaraju GM, Bunker RD, Cavadini S, et al. Crystal structure of the human COP9 signalosome. Nature 2014; 512:161165.

100 Sobhian B, Shao G, Lilli DR, et al. RAP80 targets BRCA1 
to specific ubiquitin structures at DNA damage sites. Science 2007; 316:1198-1202.

101 Yao T, Cohen RE. A cryptic protease couples deubiquitination and degradation by the proteasome. Nature 2002; 419:403407.

102 McCullough J, Row PE, Lorenzo O, et al. Activation of the endosome-associated ubiquitin isopeptidase AMSH by STAM, a component of the multivesicular body-sorting machinery. Curr Biol 2006; 16:160-165.

103 Shrestha RK, Ronau JA, Davies CW, et al. Insights into the mechanism of deubiquitination by JAMM deubiquitinases from cocrystal structures of the enzyme with the substrate and product. Biochemistry 2014; 53:3199-3217.

104 Bueno AN, Shrestha RK, Ronau JA, et al. Dynamics of an active-site flap contributes to catalysis in a JAMM family metallo deubiquitinase. Biochemistry 2015; 54:6038-6051.

105 Davies CW, Paul LN, Das C. Mechanism of recruitment and activation of the endosome-associated deubiquitinase AMSH. Biochemistry 2013; 52:7818-7829.

106 Kim MS, Kim JA, Song HK, Jeon H. STAM-AMSH interaction facilitates the deubiquitination activity in the C-terminal AMSH. Biochem Biophys Res Commun 2006; 351:612-618.

107 Wei N, Deng XW. The COP9 signalosome. Annu Rev Cell Dev Biol 2003; 19:261-286.

108 Lyapina S, Cope G, Shevchenko A, et al. Promotion of NEDD-CUL1 conjugate cleavage by COP9 signalosome. Science 2001; 292:1382-1385.

109 Schwechheimer C, Serino G, Callis J, et al. Interactions of the COP9 signalosome with the E3 ubiquitin ligase SCFTIRI in mediating auxin response. Science 2001; 292:1379-1382.

110 Cope GA, Suh GS, Aravind L, et al. Role of predicted metalloprotease motif of Jab1/Csn5 in cleavage of Nedd8 from Cul1. Science 2002; 298:608-611.

111 Sharon M, Mao H, Boeri Erba E, Stephens E, Zheng N, Robinson CV. Symmetrical modularity of the COP9 signalosome complex suggests its multifunctionality. Structure 2009; 17:31-40.

112 Tomko RJ Jr, Hochstrasser M. Molecular architecture and assembly of the eukaryotic proteasome. Annu Rev Biochem 2013; 82:415-445.

113 Tomko RJ Jr, Taylor DW, Chen ZA, Wang HW, Rappsilber J, Hochstrasser M. A single alpha helix drives extensive remodeling of the proteasome lid and completion of regulatory particle assembly. Cell 2015; 163:432-444.

114 Mansour W, Nakasone MA, von Delbruck M, et al. Disassembly of Lys11 and mixed linkage polyubiquitin conjugates provides insights into function of proteasomal deubiquitinases Rpn11 and Ubp6. J Biol Chem 2015; 290:4688-4704.

115 Pathare GR, Nagy I, Sledz P, et al. Crystal structure of the proteasomal deubiquitylation module Rpn8-Rpn11. Proc Natl Acad Sci USA 2014; 111:2984-2989.

116 Worden EJ, Padovani C, Martin A. Structure of the Rpn11Rpn8 dimer reveals mechanisms of substrate deubiquitination during proteasomal degradation. Nat Struct Mol Biol 2014; 21:220-227.

117 Dambacher CM, Worden EJ, Herzik MA, Martin A, Lander GC. Atomic structure of the $26 \mathrm{~S}$ proteasome lid reveals the mechanism of deubiquitinase inhibition. Elife 2016; 5:e13027.

118 Ye Y, Scheel H, Hofmann K, Komander D. Dissection of USP catalytic domains reveals five common insertion points. $\mathrm{Mol}$ Biosyst 2009; 5:1797-1808.

119 Lima CD, Reverter D. Structure of the human SENP7 catalytic domain and poly-SUMO deconjugation activities for SENP6 and SENP7. J Biol Chem 2008; 283:32045-32055.

120 Alegre KO, Reverter D. Structural insights into the SENP6 Loop1 structure in complex with SUMO2. Protein Sci 2014; 23:433-441.

121 Hepowit NL, Uthandi S, Miranda HV, et al. Archaeal JAB1/ MPN/MOV34 metalloenzyme (HvJAMM1) cleaves ubiquitin-like small archaeal modifier proteins (SAMPs) from protein-conjugates. Mol Microbiol 2012; 86:971-987.

122 Balakirev MY, Jaquinod M, Haas AL, Chroboczek J. Deubiquitinating function of adenovirus proteinase. J Virol 2002; 76:6323-6331.

123 Perrett CA, Lin DY, Zhou D. Interactions of bacterial proteins with host eukaryotic ubiquitin pathways. Front Microbiol 2011; 2:143.

124 Beckmann JF, Fallon AM. Detection of the Wolbachia protein WPIP0282 in mosquito spermathecae: implications for cytoplasmic incompatibility. Insect Biochem Mol Biol 2013; 43:867-878.

125 Catic A, Misaghi S, Korbel GA, Ploegh HL. ElaD, a deubiquitinating protease expressed by E. coli. PLoS One 2007; 2:e381.

$126 \mathrm{Li} \mathrm{SJ}$, Hochstrasser M. A new protease required for cell-cycle progression in yeast. Nature 1999; 398:246-251.

127 Bardill JP, Miller JL, Vogel JP. IcmS-dependent translocation of SdeA into macrophages by the Legionella pneumophila type IV secretion system. Mol Microbiol 2005; 56:90-103.

128 Furtado AR, Essid M, Perrinet S, et al. The chlamydial OTU domain-containing protein ChlaOTU is an early type III secretion effector targeting ubiquitin and NDP52. Cell Microbiol 2013; 15:2064-2079.

129 Hotson A, Chosed R, Shu H, Orth K, Mudgett MB. Xanthomonas type III effector XopD targets SUMO-conjugated proteins in planta. Mol Microbiol 2003; 50:377-389.

130 Claessen JH, Witte MD, Yoder NC, Zhu AY, Spooner E, Ploegh HL. Catch-and-release probes applied to semi-intact cells reveal ubiquitin-specific protease expression in Chlamydia trachomatis infection. Chembiochem 2013; 14:343352.

131 Misaghi S, Balsara ZR, Catic A, Spooner E, Ploegh HL, Starnbach MN. Chlamydia trachomatis-derived deubiquitinating enzymes in mammalian cells during infection. Mol Microbiol 2006; 61:142-150.

132 Penz T, Schmitz-Esser S, Kelly SE, et al. Comparative genomics suggests an independent origin of cytoplasmic incompatibility in Cardinium hertigii. PLoS Genet 2012; 8:e1003012.

133 Havey JC, Roy CR. Toxicity and SidJ-mediated suppression of toxicity require distinct regions in the SidE family of Legionella pneumophila effectors. Infect Immun 2015; 83:35063514.

134 Rytkonen A, Poh J, Garmendia J, et al. SseL, a Salmonella deubiquitinase required for macrophage killing and virulence. Proc Natl Acad Sci USA 2007; 104:3502-3507.

135 Noel L, Thieme F, Nennstiel D, Bonas U. Two novel type III-secreted proteins of Xanthomonas campestris pv. vesicato- 
ria are encoded within the hrp pathogenicity island. J Bacteriol 2002; 184:1340-1348.

136 Schlieker C, Korbel GA, Kattenhorn LM, Ploegh HL. A deubiquitinating activity is conserved in the large tegument protein of the herpesviridae. J Virol 2005; 79:15582-15585.

137 Chenon M, Camborde L, Cheminant S, Jupin I. A viral deubiquitylating enzyme targets viral RNA-dependent RNA polymerase and affects viral infectivity. EMBO J 2012; 31:741753.

138 Chosed R, Tomchick DR, Brautigam CA, et al. Structural analysis of Xanthomonas XopD provides insights into substrate specificity of ubiquitin-like protein proteases. $J$ Biol Chem 2007; 282:6773-6782.

139 McGrath WJ, Ding J, Didwania A, Sweet RM, Mangel WF. Crystallographic structure at $1.6-\AA$ resolution of the human adenovirus proteinase in a covalent complex with its 11-amino-acid peptide cofactor: insights on a new fold. Biochim Biophys Acta 2003; 1648:1-11.

140 Mangel WF, Baniecki ML, McGrath WJ. Specific interactions of the adenovirus proteinase with the viral DNA, an 11-amino-acid viral peptide, and the cellular protein actin. Cell Mol Life Sci 2003; 60:2347-2355.

141 Mangel WF, McGrath WJ, Toledo DL, Anderson CW. Viral DNA and a viral peptide can act as cofactors of adenovirus virion proteinase activity. Nature 1993; 361:274-275.

142 Frias-Staheli N, Giannakopoulos NV, Kikkert M, et al. Ovarian tumor domain-containing viral proteases evade ubiquitinand ISG15-dependent innate immune responses. Cell Host Microbe 2007; 2:404-416.

143 Akutsu M, Ye Y, Virdee S, Chin JW, Komander D. Molecular basis for ubiquitin and ISG15 cross-reactivity in viral ovarian tumor domains. Proc Natl Acad Sci USA 2011; 108:22282233.

144 James TW, Frias-Staheli N, Bacik JP, et al. Structural basis for the removal of ubiquitin and interferon-stimulated gene 15 by a viral ovarian tumor domain-containing protease. Proc Natl Acad Sci USA 2011; 108:2222-2227.

145 van Kasteren PB, Bailey-Elkin BA, James TW, et al. Deubiquitinase function of arterivirus papain-like protease 2 suppresses the innate immune response in infected host cells. Proc Natl Acad Sci USA 2013; 110:E838-847.

146 Whitby FG, Xia G, Pickart CM, Hill CP. Crystal structure of the human ubiquitin-like protein NEDD8 and interactions with ubiquitin pathway enzymes. J Biol Chem 1998; 273:34983-34991.

147 Huang WC, Ko TP, Li SS, Wang AH. Crystal structures of the human SUMO-2 protein at $1.6 \AA$ and $1.2 \AA$ resolution: implication on the functional differences of SUMO proteins. Eur $J$ Biochem 2004; 271:4114-4122.

148 Narasimhan J, Wang M, Fu Z, Klein JM, Haas AL, Kim JJ. Crystal structure of the interferon-induced ubiquitin-like protein ISG15. J Biol Chem 2005; 280:27356-27365.

149 Kumeta H, Watanabe M, Nakatogawa H, et al. The NMR structure of the autophagy-related protein Atg8. J Biomol NMR 2010; 47:237-241.

150 Johnston SC, Larsen CN, Cook WJ, Wilkinson KD, Hill CP. Crystal structure of a deubiquitinating enzyme (human UCH-L3) at 1.8 A resolution. EMBO J 1997; 16:3787-3796.

(c) (1) ()) $\Theta$ This work is licensed under a Creative Commons Attribution-NonCommercial-NoDerivs 4.0 Unported License. The images or other third party material in this article are included in the article's Creative Commons license, unless indicated otherwise in the credit line; if the material is not included under the Creative Commons license, users will need to obtain permission from the license holder to reproduce the material. To view a copy of this license, visit http://creativecommons.org/licenses/by-nc-nd/4.0/ 